\title{
A UNIFIED APPROACH TO GENERALIZED INVERSES OF LINEAR OPERATORS: I. ALGEBRAIC, TOPOLOGICAL AND PROJECTIONAL PROPERTIES
}

\author{
BY M. Z. NASHED ${ }^{1}$ AND G. F. VOTRUBA
}

Communicated by Robert Bartle, December 12, 1973

0. Introduction. In this announcement we abstract a theory for generalized inverses (G.I.) of a linear operator between two algebraic linear spaces, or topological vector spaces. Our approach includes as special cases all previously published approaches to G.I.'s of matrices and linear operators in Hilbert spaces. In addition it provides new results for G.I.'s in the case of normed spaces, and develops the first treatment of G.I.'s of arbitrary linear operators between two topological vector spaces. The explicit transformation of G.I.'s under changes of projectors is new even in the case of matrices. For a survey of various definitions and results on G.I.'s of linear operators, see Nashed [7] and the references cited therein; see also the papers by Erdelyi [3], Erdelyi and Ben-Israel [4], Deutsch [2], and Koliha [6], which appeared after [7]. Other recent references are cited in [8].

The proofs of these results and a detailed account of the various special cases alluded to will appear elsewhere [8].

1. Algebraic and projectional properties. Let $V$ and $W$ be vector spaces over the same (real or complex) field, and let $L$ be a linear map from $V$ into $W$. The range and null spaces of $L$ are denoted by $\mathscr{R}(L)$ and $\mathscr{N}(L)$ respectively. A linear map $M$ from $W$ into $V$ such that $L M L=L$ is called a partial (or inner) inverse of $L$. Every linear map has a partial inverse. If $M$ is a partial inverse of $L$, then $L M$ and $M L$ are idempotent, $\mathscr{R}(M L) \subset$ $\mathscr{R}(M), \mathscr{R}(L M)=\mathscr{R}(L), \mathscr{N}(M) \subset \mathscr{N}(L M)$, and $\mathscr{N}(M L) \subset \mathscr{N}(L)$. A partial inverse $M$ determines a particular complementary subspace to

AMS (MOS) subject classifications (1970). Primary 47A99, 47A50; Secondary 15A05, $41 \mathrm{~A} 65$.

Key words and phrases. Generalized inverses, projectors, domain decomposability, topological complements.

${ }^{1}$ Research of the first author was sponsored by the U.S. Army under Contract No. DA-31-124-ARO-D-462. 
$\mathscr{N}(L)$ in $V$, and a particular complementary subspace to $\mathscr{R}(L)$ in $W$; in fact we have the following algebraic direct sum decompositions:

$$
V=\mathscr{N}(L)+\mathscr{R}(M L) \text { and } W=\mathscr{R}(L)+\mathscr{N}(L M) \text {. }
$$

Conversely, we next show that selecting different complementary subspaces gives rise to different partial inverses and we relate these partial inverses to each other. In order to do this, we first characterize all of the linear idempotents whose range is a given subspace $S$ of a vector space $V$ (see also Sobczyk [9]). Let $\Lambda(V)$ denote the set of all linear maps from $V$ into $V$, let

and

$$
\mathscr{P}_{S}=\left\{P \in \Lambda(V): P^{2}=P \text { and } \mathscr{R}(P)=S\right\},
$$

$$
\mathscr{A}_{S}=\{A \in \Lambda(V): \mathscr{R}(A) \subset S \subset \mathscr{N}(A)\} .
$$

Proposition 1.1. The set $\mathscr{P}_{S}$ is an affine manifold, and the subspace parallel to $\mathscr{P}_{S}$ is $\mathscr{A}_{S}$.

THEOREM 1.2. Let $M$ be a partial inverse of $L$ and write $P=I-M L$ and $Q=L M$, where $I$ is the identity map. Let $P^{\prime}$ and $Q^{\prime}$ be linear idempotents with $\mathscr{R}\left(P^{\prime}\right)=\mathscr{N}(L)$ and $\mathscr{R}\left(Q^{\prime}\right)=\mathscr{R}(L)$. Then

$$
\begin{aligned}
M^{\prime} & =\left(I+P-P^{\prime}\right) M\left(I-Q+Q^{\prime}\right) \\
& =\left(2 I-M L-P^{\prime}\right) M\left(2 I-L M+Q^{\prime}\right)
\end{aligned}
$$

is a partial inverse of $L$ which satisfies $I-M^{\prime} L=P^{\prime}$ and $L M^{\prime}=Q^{\prime}$. On the other hand, if $M$ and $M^{\prime}$ are two partial inverses of $L$, then $\left(M-M^{\prime}\right) L \in$ $\mathscr{A}_{\mathscr{N}(L)}$ and $L\left(M^{\prime}-M\right) \in \mathscr{A}_{\mathscr{R}(L)}$. Also, $M-M L M$ is invariant under change of projectors.

We next consider the relation $M L M=M$. A linear map $M: W \rightarrow V$ which satisfies this relation is called an outer inverse of $L$. Every linear map has a nonzero outer inverse, and each such inverse $M$ induces the following decompositions: $V=\mathscr{R}(M)+\mathscr{N}(M L), W=\mathscr{N}(M)+\mathscr{R}(L M)$.

If $M$ is both an inner inverse and an outer inverse of $L$, then $L$ and $M$ are called algebraic generalized inverses (A.G.I.) of each other. In this case $V=\mathscr{N}(L)+\mathscr{R}(M)$ and $W=\mathscr{R}(L)+\mathscr{N}(M)$. Every linear map has an A.G.I.; in particular if $M$ is any partial inverse then $M L M$ is an A.G.I.

Let $P$ and $Q$ be the (algebraic) projectors corresponding to the algebraic direct sum decompositions $V=\mathscr{N}(L)+\mathscr{M}$ and $W=\mathscr{R}(L)+\mathscr{S}$, respectively. In other words, $P$ and $Q$ are linear idempotent maps with $\mathscr{R}(P)=$ $\mathscr{N}(L), \mathscr{N}(P)=\mathscr{M}, \mathscr{R}(Q)=\mathscr{R}(L)$ and $\mathscr{N}(Q)=\mathscr{S}$. Also, let $i$ and $j$ denote injections $i: \mathscr{M} \rightarrow V$ and $j: \mathscr{R}(L) \rightarrow W$. Then there exists a unique A.G.I. of $L$ (with respect to the choice of $P$ and $Q$ ), denoted by $L_{P, Q}^{\#}$. 
The construction is illustrated by the following commutative diagram:

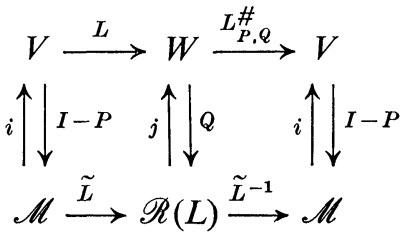

( $\backsim$ designates the restriction of an operator to the indicated domain.)

The following two questions arise naturally in this context:

(a) If we change $P$ and $Q$ to some new (algebraic) projectors $P^{\prime}=P+A$ and $Q^{\prime}=Q+B$, where $\mathscr{R}\left(P^{\prime}\right)=\mathscr{R}(P)=\mathscr{N}(L)$, and $\mathscr{R}(Q)=\mathscr{R}\left(Q^{\prime}\right)=\mathscr{R}(L)$, how is $L_{P^{\prime}, Q^{\prime}}^{\#}$ related to $L_{P, Q}^{\#}$ ?

(b) Given two A.G.I.'s $L^{\#}$ and $L^{\Uparrow}$, they induce four linear idempotent maps $Q=L L^{\#}, P=I-L^{\#} L, Q^{\prime}=L L^{\rrbracket}$, and $P^{\prime}=I-L^{\Uparrow} L$. How are the pairs $\{P, Q\}$ and $\left\{P^{\prime}, Q^{\prime}\right\}$ related?

These questions are answered in the following theorem which plays a central role in unifying the various approaches to generalized inverses in the algebraic context, as well as in the topological considerations of $\S 2$.

TheOREM 1.3. (a) Given $P=P^{2}: V \rightarrow \mathscr{N}(L)$ and $Q=Q^{2}: W \rightarrow \mathscr{R}(L)$, there is a unique solution $M$ of the system $L X=Q, X L=I-P$, and $X L X=X$. This solution is an A.G.I. of L. Any other A.G.I. (with respect to $P^{\prime}$ and $Q^{\prime}$ ) is given by $L_{P^{\prime}, Q^{\prime}}^{\#}=\left(2 I-M L-P^{\prime}\right) M\left(2 I-L M+Q^{\prime}\right)$.

(b) Using the notation of question (b) above, the following relations hold: $P^{\prime}=P+A$, and $Q^{\prime}=Q+B$, where $A \in \mathscr{A}_{\mathscr{N}(L)}$ and $B \in \mathscr{A}_{\mathscr{R}(L)}$; equivalently $P^{\prime}=P+\left(L^{\#}-L^{\top}\right) L$ and $Q^{\prime}=Q+L\left(L^{\top}-L^{\#}\right)$.

2. Topological considerations (in Hausdorff spaces). We first replace the algebraic vector space $W$ by a topological vector space $Y$. Consider $L \in \Lambda(V, Y)$ with the property that the closure of the range $(\mathscr{R}(L))^{-}$has a topological complement in $Y$, and let $Q$ be a (continuous) projector whose range is $(\mathscr{R}(L))^{-}$. Write $\mathscr{D}(M)=\mathscr{R}(L) \oplus \mathscr{N}(Q)$ and consider $L$ as being in $\Lambda(V, \mathscr{D}(M))$. We take $M$ to be a partial inverse of $L$ which satisfies $L M=Q$ on $\mathscr{D}(M)=\mathscr{R}(L) \oplus \mathscr{N}(Q)$. This can be done by Theorem 1.2. We call such an $M$ a right-topological partial inverse (abbreviated as R-T.P.I.) of $L$, and we use the notation $M=L_{r}^{\dagger}=L_{r, Q}^{\dagger}$. We consider $L_{r}^{\dagger}$ as a transformation whose domain $\mathscr{D}(M)=\mathscr{R}(L) \oplus \mathscr{N}(Q)$ is contained in the space $Y$, and point out that the domain depends on the choice of the projector $Q$ whose range is $(\mathscr{R}(L))^{-}$unless $L$ is an operator whose range is closed in $Y$. 
THEOREM 2.1. Let $L \in \Lambda(V, Y)$ have the property that $(\mathscr{R}(L))^{-}$has a topological complement, and let $Q$ and $Q^{\prime}$ be two (continuous) projectors with range $(\mathscr{R}(L))^{-}$. Let $M=L_{r, Q}^{\dagger}$ and $M^{\prime}=L_{r, Q^{\prime}}^{\dagger}$ be two corresponding R-T.P.I.'s.

(a) $\mathscr{D}(M)=\mathscr{D}\left(M^{\prime}\right)$ if and only if $\mathscr{R}\left(Q^{\prime}-Q\right) \subset \mathscr{R}(L)$.

(b) If $\mathscr{R}(L)$ is closed then every $R-T . P . I$. is defined on all of $Y$.

(c) If $\mathscr{R}(L)$ is not closed, and if $Y$ is Hausdorff and locally convex, then for any $y_{0} \notin(\mathscr{R}(L))^{-}$we can select $Q$ and $Q^{\prime}$ so that $y_{0} \in \mathscr{D}(M)$ and $y_{0} \notin \mathscr{D}\left(M^{\prime}\right)$.

The case of a left-topological partial inverse is more complicated. We consider here a linear operator $L$ whose domain $\mathscr{D}(L)$ lies in a topological vector space $X$ and whose range is considered to be in an (algebraic) vector space $W$. Here $\mathscr{D}(L)$ is not necessarily dense in $X$. We introduce a notion of decomposability which is weaker than a related notion used in Hilbert spaces by Hestenes [5], Arghiriade [1] and others.

Definition 2.2. Let $\mathscr{D}(L) \subset X$. We say $L$ is decomposable with respect to the projector $P \in \mathscr{L}(X)$, the space of continuous operators on $X$, if $\mathscr{N}^{\prime}(L) \subset \mathscr{R}(P)$ and if $P x \in \mathscr{N}(L)$ for all $x \in \mathscr{D}(L)$. In this case we call

$$
\mathscr{C}_{P}(L)=\mathscr{D}(L) \cap \mathscr{N}(P)
$$

the carrier of $L$ with respect to $P$.

We point out here that $L$ may be decomposable with respect to one projector but not with respect to another projector whose range contains $\mathscr{N}(L)$. In particular if $\mathscr{D}(L)$ is dense in a Hilbert space $H$, it may be the case that $L$ is not decomposable with respect to the orthogonal (selfadjoint) projector but that $L$ is decomposable with respect to some other (nonorthogonal) projector.

DeFInITION 2.3. If $\mathscr{D}(L) \subset X$ and $L$, considered as being in $\Lambda(\mathscr{D}(L), W)$, has a partial inverse $M$ with the property that $M L$ has an extension to a projector $I-P \in \mathscr{L}(X)$ with $\mathscr{R}(P) \supset(\mathscr{N}(T))^{-}$(the closure being taken in $X$ ), we say that $M$ is a left-topological partial inverse of $L$ (abbreviated as L-T.P.I.). We will use the notation $M=L_{l}^{\dagger}=L_{l, P}^{\dagger}$ to denote a L-T.P.I.

THEOREM 2.4. If $M=L_{l, P^{\prime}}^{\dagger}$ then $L$ is decomposable with respect to $P, \mathscr{D}(L)=\mathscr{N}(L) \oplus \mathscr{C}_{P}(L)$, and $L$ can be extended to an operator $\hat{L}$ with a closed null space which also has $M$ as a L-T.P.I. If $\mathscr{R}(I-P)=(\mathscr{R}(M L))$, then $\hat{L}$ is densely defined.

TheOREM 2.5. If $\mathscr{D}(L) \subset X$ and $L$ is decomposable with respect to $P$, then L has a L-T.P.I.

We finally get to the case where $T$ is a linear operator whose domain lies in a topological vector space $X$ and whose range lies in a topological vector space $Y$. 
DEFINITION 2.6. If $U$ is both a L-T.P.I. and a R-T.P.I. of $T$, and $U T U=$ $U$, we say that $U$ is a topological generalized inverse (T.G.I.) of $T$, and we write $U=T^{\dagger}=T_{P, Q}^{\dagger}$.

THEOREM 2.7. If $T$ is decomposable with respect to $P$ and if there exists a (continuous) projector $Q$ onto $(\mathscr{R}(T))^{-}$, then $T$ has a T.G.I. $T^{\dagger}=T_{P, Q}^{\dagger}$ which satisfies

$$
\mathscr{D}\left(T^{\dagger}\right)=\mathscr{R}(T) \oplus \mathscr{N}(Q), \quad \mathscr{R}\left(T^{\dagger}\right)=\mathscr{C}_{P}(T), \quad \mathscr{N}\left(T^{\dagger}\right)=\mathscr{N}(Q),
$$

$T^{\dagger} T x=x-P x$ for $x \in \mathscr{D}(T)$, and $T T^{\dagger} y=Q y$ for all $y \in \mathscr{D}\left(T^{\dagger}\right)$.

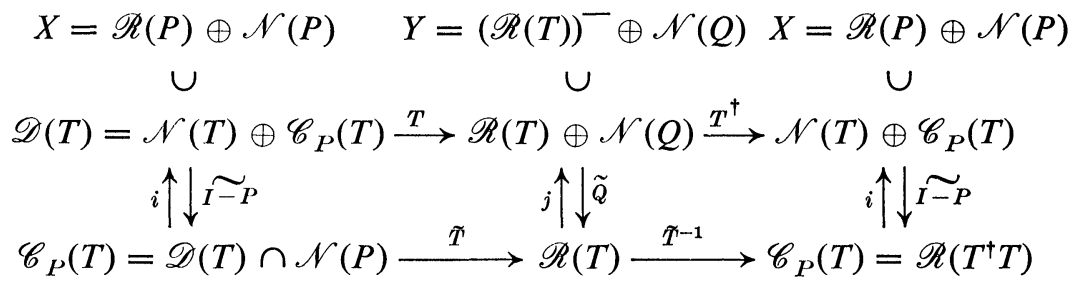

The next theorem gives conditions that will yield a continuous generalized inverse. Recall that a continuous linear operator $T: X \rightarrow Y$ with $\mathscr{D}(T)=X$ is a topological homomorphism if the image of every open set in $X$ is open in $T(X)$ in the induced topology.

THEOREM 2.8. If $T: X \rightarrow Y$ is a topological homomorphism whose null space has a topological complement in $X$ and whose range has a topological complement in $Y$, then $T$ has a generalized inverse (defined on all of $Y$ ) which is itself a topological homomorphism.

COROLlaRY 2.9. If $X$ and $Y$ are complete metrizable topological vector spaces, then any $T \in \mathscr{L}(X, Y)$ such that $\mathscr{N}(T)$ is complemented in $X$ and $\mathscr{R}(T)$ is complemented in $Y$ has a generalized inverse which is a topological homomorphism.

\section{REFERENCES}

1. E. Arghiriade, Sur l'inverse généralisée d'un opérateur linéaire, dans les espaces de Hilbert, Atti Accad. Naz. Lincei Rend. Cl. Sci. Fis. Mat. Natur. (8) 45 (1968), 471-477. MR 40 \#706.

2. E. Deutsch, Semi-inverses, reflexive semi-inverses, and pseudoinverses of an arbitrary linear transformation, Linear Algebra and Appl. 4 (1971), 313-322. MR 44 \#5325.

3. I. Erdelyi, A generalized inverse for arbitrary operators between Hilbert spaces, Proc. Cambridge Philos. Soc. 71 (1972), 43-50.

4. I. Erdelyi and A. Ben-Israel, Extremal solutions of linear equations and generalized inversion between Hilbert spaces, J. Math. Anal. Appl. 39 (1972), 298-313. MR 46 \#9778.

5. M. R. Hestenes, Relative self-adjoint operators in Hilbert spaces, Pacific J. Math. 11 (1961), 1315-1357. MR 25 \#456. 
6. J. J. Koliha, Power convergence and pseudoinverses of operators between Banach spaces, J. Math. Anal. Appl. (to appear); also Bull. Amer. Math. Soc. 80 (1974), 325-328.

7. M. Z. Nashed, Generalized inverses, normal solvability, and iteration for singular operator equations, Nonlinear Functional Anal. and Appl. (Proc Advanced Sem., Math. Res. Center, Univ. of Wisconsin, Madison, Wis., 1970), Academic Press, New York, 1971, pp. 311-359. MR 43 \#1003.

8. M. Z. Nashed and G. F. Votruba, A unifying theory for generalized inverses of linear operators, Proc. Conf. Generalized Inverses and Application, M. Z. Nashed (editor), Academic Press, New York (to appear).

9. A. Sobczyk, Projections in Minkowski and Banach spaces, Duke Math. J. 8 (1941), 78-106. MR 2, 220.

School of Mathematics, Georgia Institute of Technology, Atlanta, Georgia 30332

Department of Mathematics, University of Montana, Missoula, Montana 59801 